\title{
A Stochastic Flows Approach for Asset Allocation with Hidden Economic Environment
}

\author{
Tak Kuen Siu \\ Department of Applied Finance and Actuarial Studies, Faculty of Business and Economics, Macquarie University, \\ Sydney, NSW 2109, Australia \\ Correspondence should be addressed to Tak Kuen Siu; ktksiu2005@gmail.com
}

Received 11 August 2014; Revised 23 December 2014; Accepted 6 January 2015

Academic Editor: Huyên Pham

Copyright (C) 2015 Tak Kuen Siu. This is an open access article distributed under the Creative Commons Attribution License, which permits unrestricted use, distribution, and reproduction in any medium, provided the original work is properly cited.

\begin{abstract}
An optimal asset allocation problem for a quite general class of utility functions is discussed in a simple two-state Markovian regimeswitching model, where the appreciation rate of a risky share changes over time according to the state of a hidden economy. As usual, standard filtering theory is used to transform a financial model with hidden information into one with complete information, where a martingale approach is applied to discuss the optimal asset allocation problem. Using a martingale representation coupled with stochastic flows of diffeomorphisms for the filtering equation, the integrand in the martingale representation is identified which gives rise to an optimal portfolio strategy under some differentiability conditions.
\end{abstract}

\section{Introduction}

The optimal asset allocation problem has long been an important topic in financial economics. From the practical perspective, the problem may be of importance to investment managers in banks, financial institutions, hedge funds, insurance companies, and pension funds. The problem may also be of interest to individual investors and policyholders of defined contribution pension funds. A scientific approach to the optimal asset allocation problem was pioneered by Markowitz [1]. In the Markowitz paradigm, a single-period model was considered and the problem was formulated as a mean-variance optimization problem. Merton [2, 3] pioneered the study of the optimal asset allocation problem based on the maximization of an expected utility in a continuous-time economy. Using dynamic programming, Merton derived a Hamilton-Jacobi-Bellman (HJB) equation governing the value function of the problem under some differentiability conditions. For a particular class of utility functions, say a power utility, Merton obtained a closed-form expression for an optimal portfolio strategy which is known as the Merton ratio.

Pliska [4], Karatzas et al. [5], and Cox and Huang [6] pioneered an alternative approach to the optimal asset allocation problem in continuous time. This approach is known as the martingale approach. The key idea of the martingale approach is not unlike that of a risk-neutral valuation of a contingent claim. Firstly, an equivalent martingale measure under which the discounted optimal wealth process is a martingale is determined. Then, the integrand in a martingale representation of the discounted optimal wealth process is used to identify an optimal portfolio process. For discussions on the martingale approach, one may refer to, for example, Cvitanic and Karatzas [7], Karatzas and Shreve [8], Elliott and Kopp [9], and Pham [10]. Gerber and Shiu [11] considered an approach based on a tool used in actuarial science, namely, the Esscher transform, to discuss the optimal asset allocation problem. This approach is related to the martingale approach; see the discussion by Boyle [12].

Recently, the optimal asset allocation problem in Markovian regime-switching models has received some attention in the literature. The rationale of considering the problem in Markovian regime-switching models is to incorporate the impact of structural changes in economic conditions on price dynamics and investment decision making. Some works on the optimal asset allocation problem in Markovian regimeswitching models are in, for example, Zhou and Yin [13], Yin and Zhou [14], Sass and Haussmann [15], Baeuerle and Rieder [16], Jang et al. [17], Nagai and Runggaldier [18], Sotomayor and Cadenillas [19], Zhang et al. [20], Elliott 
and Siu [21], Elliott et al. [22], Korn et al. [23], Siu [2426], Shen and Siu [27], and others. Both situations where the modulating Markov chain is observable and where it is hidden were considered. Different approaches to stochastic optimal control such as the HJB dynamic programming approach, the martingale approach (coupled with Malliavin calculus), and the backward stochastic differential equation approach were adopted.

In this paper we study an optimal asset allocation problem for a quite general class of utility functions in a simple twostate Markovian regime-switching model. We suppose that the appreciation rate of a risky share changes over time according to the state of a hidden economy. The evolution of the two-state hidden economy over time is assumed to be governed by a continuous-time, two-state, hidden Markov chain, where the two states may be interpreted as "Expansion" and "Recession." As usual, the optimal asset allocation problem is discussed in two steps. Firstly, standard filtering theory is adopted to turn an economy with hidden information into one with complete information. The latter is called a "filtered" economy and is complete. That is, in the "filtered" economy, there is a unique equivalent martingale measure. A martingale approach is then used to discuss the optimal asset allocation problem in the "filtered" economy. Using a martingale representation coupled with stochastic flows of diffeomorphisms for the filtering equation, the integrand in the martingale representation is identified which gives rise to an optimal portfolio strategy under some differentiability conditions. A partial differential equation for the optimal wealth conditioning on the values of the underlying state variables is also obtained. This may be called an optimal wealth function which characterises a functional relationship between the current optimal wealth and the current values of the underlying state variables. The approach adopted in this paper can be used in a general $N$-state case. However, it seems that, in the two-state case, the results of the filters and the partial differential equation for the optimal wealth are neater than those arising from the $N$-state case. Indeed, the two-state case may not be without practical relevance. Taylor $[28,29]$ provided some discussions on the practical relevance of using a two-state Markovian regime-switching process for modeling financial returns.

The rest of the paper is structured as follows. The next section presents the model dynamics in the original economy with hidden information and in the "filtered" economy. In Section 3, the optimal asset allocation problem in the "filtered" economy is presented and the martingale approach is used for solving the problem. The use of stochastic flows to identify the integrand in the martingale representation in the filtered market is then presented in Section 4. The final section gives a summary and suggests some possible topics for further research.

\section{Model Dynamics and Filtering}

The modelling and filtering frameworks presented here are standard in the literature on optimal asset allocation in a hidden Markovian regime-switching model; see, for example,
Siu [24-26], Korn et al. [23], Wei et al. [30], and Elliott and Siu [21], amongst others.

A continuous-time economy with two primitive securities, namely, a bond and a risky share, is considered. As usual, uncertainty in the economy is described by a complete probability space $(\Omega, \mathscr{F}, \mathbb{P})$, where $\mathbb{P}$ is a real-world probability measure. The time parameter set of the economy is given by a finite time horizon $\mathscr{T}:=[0, T]$, where $T<\infty$. The evolution of the state of a hidden economy over time is modeled by a continuous-time, two-state, hidden Markov chain $\{\mathbf{X}(t) \mid t \in \mathscr{T}\}$ on $(\Omega, \mathscr{F}, \mathbb{P})$. The state space of the chain is taken to be $\left\{\mathbf{e}_{1}, \mathbf{e}_{2}\right\}$, where $\mathbf{e}_{1}=(1,0)^{\prime} \in \mathfrak{R}^{2}$ and $\mathbf{e}_{2}=(0,1)^{\prime} \in \mathfrak{R}^{2} \cdot \mathbf{y}^{\prime}$ is the transpose of a vector or a matrix $\mathbf{y}$. The state space $\left\{\mathbf{e}_{1}, \mathbf{e}_{2}\right\}$ is called the canonical state space of the chain and was adopted in Elliott et al. [31]. The states " $\mathbf{e}_{1}$ " and " $\mathbf{e}_{2}$ " may be interpreted as "Expansion" and "Recession" of an economy, respectively. As usual, the probability laws of the chain are specified by its intensity matrix, or rate matrix, which is defined as

$$
\mathbf{A}:=\left[\begin{array}{cc}
-a_{1} & a_{1} \\
a_{2} & -a_{2}
\end{array}\right],
$$

where $a_{1}, a_{2}>0$.

A two-state Markovian regime-switching model for asset price dynamics may be justified both theoretically and empirically (see Taylor [28, 32]). Indeed, Taylor [29] pointed out that a two-state Markov chain is sufficient to distinguish a good economy from an economy experiencing distresses.

Let $r$ be the constant continuously compounded rate of interest of the bond, where $r>0$. Then the evolution of the bond price over time is governed by

$$
B(t)=e^{r t}, \quad B(0)=1 .
$$

For each $t \in \mathscr{T}$, let $\mu(t)$ be the appreciation rate of the risky share at time $t$. Again it is supposed that $\mu(t)$ is modulated by the chain as

$$
\mu(t)=\langle\boldsymbol{\mu}, \mathbf{X}(t)\rangle,
$$

where $\boldsymbol{\mu}:=\left(\mu_{1}, \mu_{2}\right)^{\prime} \in \mathfrak{R}^{2} ; \mu_{1}$ and $\mu_{2}$ are the appreciation rates of the risky share when the economy is in an expansion and when it is in a recession, respectively, where $\mu_{1}>\mu_{2}$; $\langle\cdot, \cdot\rangle$ is the scalar product in $\Re^{2}$; since the chain $\{\mathbf{X}(t) \mid t \in$ $\mathscr{T}\}$ is hidden, the appreciation rate $\mu(t)$ defined as above is unobservable.

Let $\sigma$ be the constant volatility of the risky share, where $\sigma>0$, and $\{W(t) \mid t \in \mathscr{T}\}$ is a standard Brownian motion on $(\Omega, \mathscr{F}, \mathbb{P})$. Then we suppose that under $\mathbb{P}$ the evolution of the price of the risky share over time is governed by

$$
d S(t)=\mu(t) S(t)+\sigma S(t) d W(t), \quad S(0)=s_{0} \in(0, \infty) .
$$

Note that if one considers a general situation where the volatility is modulated by the hidden Markov chain as well, one may need to take into account some potential issues. Firstly, standard filtering theory may not be conveniently used to turn the economy with hidden states into one with 
observable states. Secondly, since $\sigma$ is completely identified by the predictable quadratic variation of the price process of the risky share, it should be observable. Thirdly, an exact, finitedimensional filtering equation for the hidden Markov chain may be difficult, if not impossible, to derive if the volatility is also modulated by the hidden Markov chain. One may refer to, for example, Guo [33], Gerber and Shiu [34], Siu [24], and Elliott and Siu [21], for related discussions.

Note that the price process $\{S(t) \mid t \in \mathscr{T}\}$ of the risky share is observable. However, both the drift process $\{\mu(t) \mid t \in \mathscr{T}\}$ and the Brownian motion $\{W(t) \mid t \in \mathscr{T}\}$ are unobservable.

In what follows, we will adopt standard filtering theory to turn the economy with hidden states into one with observable states.

Firstly the information structure is specified. Let $\mathbb{F}:=$ $\{\mathscr{F}(t) \mid t \in \mathscr{T}\}$ be the right-continuous and $\mathbb{P}$-complete filtration generated by the price process $\{S(t) \mid t \in \mathscr{T}\}$ of the risky share. This describes the flow of observable market information. Let $\mathbb{F}^{\mathbf{X}}:=\left\{\mathscr{F}^{\mathbf{X}}(t) \mid t \in \mathscr{T}\right\}$ be the rightcontinuous, $\mathbb{P}$-complete filtration generated by the chain $\mathbf{X}$. For each $t \in \mathscr{T}$, let

$$
\mathscr{G}(t):=\mathscr{F}^{\mathbf{X}}(t) \vee \mathscr{F}(t),
$$

where $\mathscr{A} \vee \mathscr{B}$ is the minimal $\sigma$-field containing both $\sigma$ fields $\mathscr{A}$ and $\mathscr{B}$. Write $\mathbb{G}:=\{\mathscr{G}(t) \mid t \in \mathscr{T}\}$.

For any integrable, $\mathbb{G}$-adapted, process, $\{\phi(t) \mid t \in \mathscr{T}\}$, let $\{\widehat{\phi}(t) \mid t \in \mathscr{T}\}$ be its $\mathbb{F}$-optional projection under $\mathbb{P}$. Then, for each $t \in \mathscr{T}$,

$$
\widehat{\phi}(t)=E[\phi(t) \mid \mathscr{F}(t)], \quad \mathbb{P} \text {-a.s. }
$$

Here $E$ is the expectation operator under $\mathbb{P}$. It is known that the optional projection takes into account the measurability in $(t, \omega) \in \mathscr{T} \times \Omega$.

Define, for each $t \in \mathscr{T}$,

$$
p(t):=\left\langle\widehat{\mathbf{X}}(t), \mathbf{e}_{1}\right\rangle=\mathbb{P}\left(\mathbf{X}(t)=\mathbf{e}_{1} \mid \mathscr{F}(t)\right) .
$$

This is the conditional, or posterior, probability that the hidden economy is in an expansion at time $t$ given the observable information up to time $t$. We suppose that $p(0)=$ $p_{0}$, where $p_{0}$ is a given constant taking a value in $(0,1)$.

Define the process $\{\widehat{W}(t) \mid t \in \mathscr{T}\}$ by putting

$$
\widehat{W}(t):=W(t)+\int_{0}^{t}\left(\frac{\mu(u)-\mu_{1} p(u)-\mu_{2}(1-p(u))}{\sigma}\right) d u .
$$

Then it was shown in Lipster and Shiryaev [35] that $\{\widehat{W}(t)$ $t \in \mathscr{T}\}$ is an $(\mathbb{F}, \mathbb{P})$-standard Brownian motion. This is called the innovations process.

Then the following lemma was due to, for example, Lipster and Shiryaev [35] and Elliott [36] (see Chapter 18 therein).
Lemma 1. Let $\rho:=\left(\mu_{1}-\mu_{2}\right) / \sigma$. Then, under $\mathbb{P}$, the conditional probability process $\{p(t) \mid t \in \mathscr{T}\}$ is governed by the following stochastic differential equation:

$$
\begin{aligned}
d p(t)= & {\left[-a_{1} p(t)+a_{2}(1-p(t))\right] d t } \\
& +\rho(1-p(t)) p(t) d \widehat{W}(t), \\
& p(0)=p_{0} \in(0,1) .
\end{aligned}
$$

Under $\mathbb{P}$, the price process $\{S(t) \mid t \in \mathscr{T}\}$ of the risky share can be expressed in terms of $\{\widehat{W}(t) \mid t \in \mathscr{T}\}$ as follows:

$$
d S(t)=\left[\mu_{2}+\left(\mu_{1}-\mu_{2}\right) p(t)\right] S(t) d t+\sigma S(t) d \widehat{W}(t) .
$$

Note that

$$
\widehat{\mu}(t)=\langle\boldsymbol{\mu}, \widehat{\mathbf{X}}(t)\rangle=\mu_{1} p(t)+\mu_{2}(1-p(t)) .
$$

Then, under $\mathbb{P}$,

$$
d S(t)=\widehat{\mu}(t) S(t) d t+\sigma S(t) d \widehat{W}(t) .
$$

This is used as the price process of the risky share in a "filtered" economy with complete observations. It is clear that the filtered economy is complete.

\section{Martingale Approach for Asset Allocation}

The aim of this section is to adapt the martingale approach to optimal asset allocation in the "filtered" economy described in the last section. The martingale approach to optimal asset allocation was pioneered by Pliska [4], Karatzas et al. [5], and Cox and Huang [6]. The mathematical basis of this approach is the martingale method for stochastic optimal control which was pioneered by Rishel [37], Duncan and Varaiya [38, 39], and Davis [40]. The martingale approach has been used to discuss optimal asset allocation problems in some filtered financial models (see, e.g., Sass and Haussmann [15], Korn et al. [23], and Siu [24] and the relevant references therein). In this section, the classical convex dual arguments in, for example, Karatzas and Shreve [8], Pham [10], and Cvitanic and Karatzas [7], will be used. Unlike the Lagrange multiplier arguments, the classical convex dual arguments do not require the change of order of differentiation and integration (The author would like to thank the referee for pointing out this.). The developments here are standard and follow those in Cvitanic and Karatzas [7] (Section 7 therein).

Recall that, in the "filtered" model, the price process of the risky share under $\mathbb{P}$ is given by

$$
d S(t)=\widehat{\mu}(t) S(t) d t+\sigma S(t) d \widehat{W}(t) .
$$

Furthermore, under $\mathbb{P}$, the filtering equation is given by

$$
\begin{aligned}
d p(t)= & {\left[-a_{1} p(t)+a_{2}(1-p(t))\right] d t } \\
& +\rho(1-p(t)) p(t) d \widehat{W}(t),
\end{aligned}
$$

where $p(0)=p_{0} \in(0,1)$. 
Let $\{\pi(t) \mid t \in \mathscr{T}\}$ be a portfolio process, where $\pi(t)$ is the amount of money invested in the risky share held at time $t$. Suppose that $\{\pi(t) \mid t \in \mathscr{T}\}$ is self-financing. Then, under $\mathbb{P}$, the wealth process $\left\{V^{\pi}(t) \mid t \in \mathscr{T}\right\}$ corresponding to the self-financing portfolio process $\{\pi(t) \mid t \in \mathscr{T}\}$ is governed by

$$
d V^{\pi}(t)=\left[r V^{\pi}(t)+\pi(t)(\widehat{\mu}(t)-r)\right] d t+\pi(t) \sigma d \widehat{W}(t) .
$$

To simplify the notation, the superscript $\pi$ is suppressed and write $V(t)$ for $V^{\pi}(t)$ unless otherwise stated. The initial wealth $V(0)=v_{0} \in(0, \infty)$.

Then, as usual, an admissible portfolio process is defined as follows.

Definition 2. A portfolio process $\{\pi(t) \mid t \in \mathscr{T}\}$ is said to be admissible with respect to the initial wealth $v_{0} \in(0, \infty)$ and the initial state $p_{0} \in(0,1)$ (i.e., $\left.\{\pi(t) \mid t \in \mathscr{T}\} \in \mathscr{A}\left(v_{0}, p_{0}\right)\right)$, if it satisfies the following conditions:

(1) $\{\pi(t) \mid t \in \mathscr{T}\}$ is self-financing;

(2) $\{\pi(t) \mid t \in \mathscr{T}\}$ is a measurable, $\mathbb{F}$-adapted process such that

$$
\int_{0}^{T}|\pi(t)|^{2} d t<\infty, \quad \mathbb{P}-\text { a.s }
$$

(3) for each $t \in \mathscr{T}, V(t):=V(t, \omega) \geq 0$, for almost all $(t, \omega) \in \mathscr{T} \times \Omega$

(4) hence

$$
\int_{0}^{T}|r V(t)+\pi(t)(\widehat{\mu}(t)-r)| d t<\infty, \quad \mathbb{P} \text {-a.s; }
$$

(5) the stochastic differential equation governing the wealth process $\{V(t) \mid t \in \mathscr{T}\}$ has a unique strong solution.

Consider now a utility function $U:(0, \infty) \rightarrow \mathfrak{R}$ such that it satisfies the following standard conditions:

(1) $U \in \mathscr{C}^{1}((0, \infty))$, where $\mathscr{C}^{1}((0, \infty))$ is the space of continuously differentiable functions on $(0, \infty)$;

(2) $U$ is strictly increasing and strictly concave;

(3) the derivative $U^{\prime}$ of $U$ is such that

$$
\lim _{v \rightarrow \infty} U^{\prime}(v)=0, \quad \lim _{v \rightarrow 0^{+}} U^{\prime}(v)=\infty
$$

Define

$$
\mathscr{A}_{1}\left(v_{0}, p_{0}\right):=\left\{\pi \in \mathscr{A}\left(v_{0}, p_{0}\right) \mid E\left[U^{-}(V(T))\right]<\infty\right\},
$$

where $U^{-}$is the negative part of $U$.

Then for each portfolio process $\pi \in \mathscr{A}_{1}\left(v_{0}, p_{0}\right)$, initial wealth $v_{0} \in(0, \infty)$, and initial information state $p_{0} \in(0,1)$, the performance functional $J\left(\pi ; v_{0}, p_{0}\right)$ is defined by

$$
J\left(\pi ; v_{0}, p_{0}\right):=E[U(V(T))] .
$$

The objective of an economic agent is to select $\pi \in \mathscr{A}_{1}\left(v_{0}, p_{0}\right)$ so as to maximize $J\left(\pi ; v_{0}, p_{0}\right)$, that is, to solve the following optimization problem:

$$
\Phi\left(v_{0}, p_{0}\right):=\sup _{\pi \in \mathscr{A}_{1}\left(v_{0}, p_{0}\right)} J\left(\pi ; v_{0}, p_{0}\right)
$$

where $\Phi\left(v_{0}, p_{0}\right)$ is the value of the optimization problem.

Let $\{\hat{\theta}(t) \mid t \in \mathscr{T}\}$ be a bounded, $\mathbb{F}$-predictable process defined by

$$
\widehat{\theta}(t):=\left(\frac{r-\mu_{1}}{\sigma}\right) p(t)+\left(\frac{r-\mu_{2}}{\sigma}\right)(1-p(t)) .
$$

Consider the $\mathbb{F}$-adapted process $\{\Lambda(t) \mid t \in \mathscr{T}\}$ defined by putting

$$
\Lambda(t):=\exp \left(\int_{0}^{t} \widehat{\theta}(u) d \widehat{W}(u)-\frac{1}{2} \int_{0}^{t} \widehat{\theta}^{2}(u) d u\right) .
$$

Since $\{\hat{\theta}(t) \mid t \in \mathscr{T}\}$ is bounded, $\{\Lambda(t) \mid t \in \mathscr{T}\}$ is an $(\mathbb{F}, \mathbb{P})$ exponential martingale. Consequently, $E[\Lambda(T)]=1$.

A new probability measure $\mathbb{Q}$ equivalent to $\mathbb{P}$ on $\mathscr{F}(T)$ can then be defined by setting

$$
\left.\frac{d \mathbb{Q}}{d \mathbb{P}}\right|_{\mathscr{F}(T)}:=\Lambda(T) .
$$

By Girsanov's theorem, the process $\left\{\widehat{W}^{\mathbb{Q}}(t) \mid t \in \mathscr{T}\right\}$ defined by

$$
\widehat{W}^{\mathbb{Q}}(t):=\widehat{W}(t)-\int_{0}^{t} \widehat{\theta}(u) d u
$$

is an $(\mathbb{F}, \mathbb{Q})$-standard Brownian motion.

Let $\{\widetilde{V}(t) \mid t \in \mathscr{T}\}$ be the discounted wealth process, where $\widetilde{V}(t):=e^{-r t} V(t)$ for each $t \in \mathscr{T}$. Then, under $\mathbb{Q}$,

$$
\widetilde{V}(t)=v_{0}+\int_{0}^{t} \pi(u) e^{-r u} \sigma d \widehat{W}^{\mathbb{Q}}(u) .
$$

This is an $(\mathbb{F}, \mathbb{Q})$-(local)-martingale. Indeed it is an $(\mathbb{F}, \mathbb{Q})$ martingale.

Note that $\pi \in \mathscr{A}_{1}\left(v_{0}, p_{0}\right) \subset \mathscr{A}\left(v_{0}, p_{0}\right)$, so $\{\widetilde{V}(t) \mid t \in \mathscr{T}\}$ is bounded from below. Consequently, using the Fatou lemma, $\{\widetilde{V}(t) \mid t \in \mathscr{T}\}$ is an $(\mathbb{F}, \mathbb{Q})$-supermartingale (see Karatzas and Shreve [8], Page 92 therein). Then

$$
E^{\mathbb{Q}}[\widetilde{V}(T)] \leq v_{0},
$$

where $E^{\mathbb{Q}}$ is the expectation operator under $\mathbb{Q}$.

Since $U$ is strictly concave, $U^{\prime}$ is strictly decreasing. Consequently, there is an inverse map $I:(0, \infty) \rightarrow(0, \infty)$ of $U^{\prime}$ which is also strictly decreasing. Furthermore, $I(0+)=\infty$ and $I(\infty)=0$. Define, for each $\gamma \in(0, \infty)$,

$$
L(\gamma):=E\left[e^{-r T} \Lambda(T) I\left(\gamma \Lambda(T) e^{-r T}\right)\right] .
$$

Suppose that, for each $\gamma \in(0, \infty), L(\gamma)<\infty$. Then the function $L:(0, \infty) \rightarrow(0, \infty)$ is continuous and is strictly decreasing such that $L(0+)=\infty$ and $L(\infty)=0$. Let $\kappa$ be 
the inverse of $L$. Define a positive, $\mathscr{F}(T)$-measurable, integrable random variable $\xi$ by putting

$$
\xi:=I\left(\kappa\left(v_{0}\right) \Lambda(T) e^{-r T}\right)
$$

Then the following lemma is a standard result and is a particular case of Lemma 7.2 in Cvitanic and Karatzas [7]. We state the result without giving the proof.

Lemma 3. The random variable $\xi$ defined in (29) satisfies

$$
\begin{gathered}
E\left[e^{-r T} \Lambda(T) \xi\right]=v_{0}, \\
E\left[U^{-}(\xi)\right]<\infty,
\end{gathered}
$$

and, for all $\pi \in \mathscr{A}_{1}\left(v_{0}, p_{0}\right)$,

$$
J\left(\pi ; v_{0}, p_{0}\right) \leq E[U(\xi)] .
$$

The following theorem is a standard result and is a particular case of Proposition 7.3 in Cvitanic and Karatzas [7]. Note that the form of an optimal portfolio process is identified in the proof of the theorem, so we present the proof following that in Cvitanic and Karatzas [7].

Theorem 4. Let $H$ be a positive, $\mathscr{F}(T)$-measurable, integrable random variable such that

$$
E\left[e^{-r T} \Lambda(T) H\right]=E^{\mathbb{Q}}\left[e^{-r T} H\right]=v_{0}<\infty .
$$

Then there exists a portfolio process $\pi$ such that $\pi \in \mathscr{A}\left(v_{0}, p_{0}\right)$ and $V^{v_{0}, \pi}(T)=H, \mathbb{P}$-a.s.

Proof. With a slight abuse of notation, we define a positive, continuous process $\{V(t) \mid t \in \mathscr{T}\}$ by putting

$$
e^{-r t} V(t)=E^{\mathbb{Q}}\left[e^{-r T} H \mid \mathscr{F}(t)\right], \quad t \in \mathscr{T} .
$$

Then, from its definition, $V(0)=v_{0}$ and $V(T)=H, \mathbb{P}$-a.s.

Define the following $(\mathbb{F}, \mathbb{Q})$-martingale $\{M(t) \mid t \in \mathscr{T}\}$ by setting

$$
\begin{aligned}
M(t) & :=E^{\mathbb{Q}}\left[e^{-r T} H \mid \mathscr{F}(t)\right] \\
& =e^{-r t} V(t)=\widetilde{V}(t), \quad t \in \mathscr{T} .
\end{aligned}
$$

By the martingale representation theorem (see, e.g., Elliott [36], Theorem 12.33 therein), there exists an $\mathbb{F}$-progressively measurable, real-valued process $\{\psi(t) \mid t \in \mathscr{T}\}$ satisfying $\int_{0}^{T}|\psi(t)|^{2} d t, \mathbb{P}$-a.s., such that

$$
M(t)=\widetilde{V}(t)=v_{0}+\int_{0}^{t} \psi(u) d \widehat{W}^{\mathbb{Q}}(t), \quad \mathbb{P} \text {-a.s. }
$$

Comparing (26) and (35), the process $\{V(t) \mid t \in \mathscr{T}\}$ is the wealth process corresponding to the portfolio process $\pi \in \mathscr{A}\left(v_{0}, p_{0}\right)$, where the portfolio process $\pi:=\{\pi(t) \mid t \in \mathscr{T}\}$ is given by

$$
\pi(t)=\frac{\psi(t)}{\sigma e^{-r t}}, \quad t \in \mathscr{T}
$$

The following corollary is a particular case of Theorem 7.4 in Cvitanic and Karatzas [7]. It is a direct consequence of Lemma 3 and Theorem 4.

Corollary 5. Suppose $\xi$ is defined in (29). Then there exists a portfolio process $\pi^{*}:=\left\{\pi^{*}(t) \mid t \in \mathscr{T}\right\}$ such that $\pi^{*} \in \mathscr{A}_{1}\left(v_{0}, p_{0}\right), V^{v_{0}, \pi^{*}}(T)=\xi$, P-a.s., and $\Phi\left(v_{0}, p_{0}\right)=$ $J\left(\pi^{*} ; v_{0}, p_{0}\right)$.

From Corollary 5, it is clear that the optimal terminal wealth is given by $\xi$ defined in (29) and the corresponding admissible optimal portfolio process is given by $\pi^{*}$, where $\pi^{*}$ is given in the proof of Theorem 4; that is,

$$
\pi^{*}(t)=\frac{\psi(t)}{\sigma e^{-r t}}, \quad t \in \mathscr{T} .
$$

Consequently to determine the optimal portfolio process $\left\{\pi^{*}(t) \mid t \in \mathscr{T}\right\}$, the integrand $\{\psi(t) \mid t \in \mathscr{T}\}$ in martingale representation (35) must be determined. In the next section the integrand $\{\psi(t) \mid t \in \mathscr{T}\}$ will be identified using the concept of stochastic flows of diffeomorphisms.

\section{Stochastic Flows and Optimal Portfolio}

In this section the stochastic flows of diffeomorphisms for the filtering equation will be first discussed. Then under some mild differentiability conditions the integrand in the martingale representation for the discounted optimal wealth process is identified which, in turn, gives rise to an expression for the optimal portfolio. The concept of stochastic flows of diffeomorphisms has been used for option pricing and hedging; see, for example, Colwell et al. [41], Colwell and Elliott [42], Elliott and Kopp [9], and Elliott et al. [43], amongst others.

For each $z \in(0,1)$, let $Z_{s, t}(z)$ be the unique, strong solution of the filtering equation under $\mathbb{P}$ for $t \geq s$ with initial condition $Z_{s, s}(z)=z$. That is,

$$
\begin{gathered}
d Z_{s, t}(z)=\left[-a_{1} Z_{s, t}(z)+a_{2}\left(1-Z_{s, t}(z)\right)\right] d t \\
+\rho\left(1-Z_{s, t}(z)\right) Z_{s, t}(z) d \widehat{W}(t), \\
Z_{s, s}(z)=z .
\end{gathered}
$$

Then using similar arguments in Kunita $[44,45]$ and Bismut [46] (see also, e.g., Elliott and Kopp [9]), there exists a flow of diffeomorphisms $z \rightarrow Z_{s, t}(z)$ corresponding to the filtering equation. Write

$$
D_{s, t}(z):=\frac{\partial Z_{s, t}(z)}{\partial z}
$$

for the derivative of the map $z \rightarrow Z_{s, t}(z)$.

Then $D_{s, t}(z)$ satisfies the following linearized stochastic differential equation:

$$
\begin{aligned}
d D_{s, t}(z)= & -\left(a_{1}+a_{2}\right) D_{s, t}(z) d t \\
& +\rho\left(1-2 Z_{s, t}(z)\right) D_{s, t}(z) d \widehat{W}(t),
\end{aligned}
$$

with initial condition $D_{s, s}(z)=1$. 
Again using similar arguments in Kunita $[44,45]$ and Bismut [46] (see also, e.g., Elliott and Kopp [9]), the inverse $D_{s, t}^{-1}(z)$ of $D_{s, t}(z)$ exists. Furthermore, $D_{s, t}^{-1}(z)$ satisfies the following equation:

$$
\begin{aligned}
d D_{s, t}^{-1}(z)= & D_{s, t}^{-1}(z)\left(a_{1}+a_{2}\right) d t \\
& -D_{s, t}^{-1}(z) \rho\left(1-2 Z_{s, t}(z)\right) d \widehat{W}(t) \\
& +D_{s, t}^{-1}(z) \rho^{2}\left(1-2 Z_{s, t}(z)\right)^{2} d t,
\end{aligned}
$$

with initial condition $D_{s, s}^{-1}(z)=1$.

Write, for each $i=1,2$,

$$
\theta_{i}:=\frac{r-\mu_{i}}{\sigma},
$$

and, for each $s, t \in \mathscr{T}$ with $s \leq t, z \in(0,1)$,

$$
\Theta_{s, t}(z):=\theta_{1} Z_{s, t}(z)+\theta_{2}\left(1-Z_{s, t}(z)\right),
$$

so that

$$
\frac{\partial \Theta_{s, t}(z)}{\partial z}=\left(\theta_{1}-\theta_{2}\right) D_{s, t}(z) .
$$
by

Consider now the stochastic exponential $\mathscr{M}_{s, t}(z)$ defined

$$
\mathscr{M}_{s, t}(z)=1+\int_{s}^{t} \Theta_{s, u}(z) \mathscr{M}_{s, u}(z) d \widehat{W}(u)
$$

Then

$$
\mathscr{M}_{s, t}(z)=\exp \left(\int_{s}^{t} \Theta_{s, u}(z) d \widehat{W}(u)-\frac{1}{2} \int_{s}^{t} \Theta_{s, u}^{2}(z) d u\right) .
$$

This is an $(\mathbb{F}, \mathbb{P})$-martingale.

The following lemma gives an expression for the derivative $\partial \mathscr{M}_{t, T}(z) / \partial z$.

Lemma 6. Consider

$$
\frac{\partial \mathscr{M}_{t, T}(z)}{\partial z}=\mathscr{M}_{t, T}(z) \int_{t}^{T}\left(\theta_{1}-\theta_{2}\right) D_{t, u}(z) d \widehat{W}^{\mathbb{Q}}(u) .
$$

Proof. Using similar arguments in Elliott et al. [43], Proposition 3.1, for example, the result can be verified by differentiation. Firstly, from the differentiability of the solution of stochastic differential equation (45),

$$
\begin{aligned}
\frac{\partial \mathscr{M}_{t, T}(z)}{\partial z}= & \int_{t}^{T}\left(\theta_{1}-\theta_{2}\right) D_{t, u}(z) \mathscr{M}_{t, u}(z) d \widehat{W}(u) \\
& +\int_{t}^{T} \Theta_{t, u}(z) \frac{\partial \mathscr{M}_{t, u}(z)}{\partial z} d \widehat{W}(u) .
\end{aligned}
$$

Then applying Itô's differentiation rule to the product

$$
\mathscr{M}_{t, T}(z) \int_{t}^{T}\left(\theta_{1}-\theta_{2}\right) D_{t, u}(z) d \widehat{W}^{\mathbb{Q}}(u)
$$

and using (45) give

$$
\begin{aligned}
& \mathscr{M}_{t, T}(z) \int_{t}^{T}\left(\theta_{1}-\theta_{2}\right) D_{t, u}(z) d \widehat{W}^{\mathbb{Q}}(u) \\
& =\int_{t}^{T} \mathscr{M}_{t, u}(z)\left(\theta_{1}-\theta_{2}\right) D_{t, u}(z)\left(d \widehat{W}(u)-\Theta_{t, u}(z) d u\right) \\
& \quad+\int_{t}^{T}\left(\mathscr{M}_{t, u}(z) \int_{t}^{u}\left(\theta_{1}-\theta_{2}\right) D_{t, s}(z) d \widehat{W}^{\mathbb{Q}}(s)\right) \\
& \quad \cdot \Theta_{t, u}(z) d \widehat{W}(u) \\
& +\int_{t}^{T} \mathscr{M}_{t, u}(z)\left(\theta_{1}-\theta_{2}\right) D_{t, u}(z) \Theta_{t, u}(z) d u \\
& =\int_{t}^{T} \mathscr{M}_{t, u}(z)\left(\theta_{1}-\theta_{2}\right) D_{t, u}(z) d \widehat{W}(u) \\
& \quad+\int_{t}^{T}\left(\mathscr{M}_{t, u}(z) \int_{t}^{u}\left(\theta_{1}-\theta_{2}\right) D_{t, s}(z) d \widehat{W}^{\mathbb{Q}}(s)\right)
\end{aligned}
$$$$
\cdot \Theta_{t, u}(z) d \widehat{W}(u) .
$$

If

$$
\frac{\partial \mathscr{M}_{t, u}(z)}{\partial z}=\mathscr{M}_{t, u}(z) \int_{t}^{u}\left(\theta_{1}-\theta_{2}\right) D_{t, s}(z) d \widehat{W}^{\mathbb{Q}}(s),
$$

then

$$
\begin{gathered}
\mathscr{M}_{t, T}(z) \int_{t}^{T}\left(\theta_{1}-\theta_{2}\right) D_{t, u}(z) d \widehat{W}^{\mathbb{Q}}(u) \\
=\int_{t}^{T} \mathscr{M}_{t, u}(z)\left(\theta_{1}-\theta_{2}\right) D_{t, u}(z) d \widehat{W}(u) \\
\quad+\int_{t}^{T} \frac{\partial \mathscr{M}_{t, u}(z)}{\partial z} \Theta_{t, u}(z) d \widehat{W}(u) .
\end{gathered}
$$

The result follows by noting that (48) has a unique solution.

Define a function $F: \mathscr{T} \times(0, \infty) \times(0,1) \rightarrow \mathfrak{R}$ by putting

$$
\begin{aligned}
F(t, y, z):=E[ & \mathscr{M}_{t, T}(z) e^{-r T} \\
& \left.\times I\left(\kappa\left(v_{0}\right) y \mathscr{M}_{t, T}(z) e^{-r T}\right) \mid \mathscr{F}(t)\right],
\end{aligned}
$$

for each $(t, y, z) \in \mathscr{T} \times(0, \infty) \times(0,1)$.

Then we have the following lemma.

Lemma 7. If $\mathscr{M}_{0, t}\left(z_{0}\right)=y$ and $Z_{0, t}\left(z_{0}\right)=z,(y, z) \in(0, \infty) \times$ $(0,1)$, then the discounted optimal wealth $e^{-r t} V^{*}(t)$ at time $t$ is given by

$$
e^{-r t} V^{*}(t)=F(t, y, z) .
$$

Proof. The proof is standard. Note that

$$
\mathscr{M}_{0, T}\left(z_{0}\right)=\mathscr{M}_{0, t}\left(z_{0}\right) \mathscr{M}_{t, T}(z) .
$$


Then by a version of Bayes' rule and the martingale property of $\left\{\mathscr{M}_{0, t}\left(z_{0}\right) \mid t \in \mathscr{T}\right\}$,

$$
\begin{aligned}
e^{-r t} V^{*}(t)= & E^{\mathbb{Q}}\left[e^{-r T} \xi \mid \mathscr{F}(t)\right] \\
= & E^{\mathbb{Q}}\left[e^{-r T} I\left(\kappa\left(v_{0}\right) \Lambda(T) e^{-r T}\right) \mid \mathscr{F}(t)\right] \\
= & E^{\mathbb{Q}}\left[e^{-r T} I\left(\kappa\left(v_{0}\right) \mathscr{M}_{0, T}\left(z_{0}\right) e^{-r T}\right) \mid \mathscr{F}(t)\right] \\
= & E\left[\mathscr{M}_{0, T}\left(z_{0}\right) e^{-r T}\right. \\
& \left.\times I\left(\kappa\left(v_{0}\right) \mathscr{M}_{0, T}\left(z_{0}\right) e^{-r T}\right) \mid \mathscr{F}(t)\right] \\
& \cdot\left(E\left[\mathscr{M}_{0, T}\left(z_{0}\right) \mid \mathscr{F}(t)\right]\right)^{-1} \\
= & E\left[\mathscr{M}_{t, T}(z) e^{-r T}\right. \\
& \left.\times I\left(\kappa\left(v_{0}\right) \mathscr{M}_{0, T}\left(z_{0}\right) e^{-r T}\right) \mid \mathscr{F}(t)\right] \\
= & E\left[\mathscr{M}_{t, T}(z) e^{-r T}\right. \\
& \left.\times I\left(\kappa\left(v_{0}\right) y \mathscr{M}_{t, T}(z) e^{-r T}\right) \mid \mathscr{F}(t)\right] .
\end{aligned}
$$

This gives the result.

For each $(t, y, z) \in \mathscr{T} \times(0, \infty) \times(0,1)$, let

$$
F^{*}(t, y, z)=\frac{F(t, y, z)}{e^{-r t}} .
$$

Then if $\mathscr{M}_{0, t}\left(z_{0}\right)=y$ and $Z_{0, t}\left(z_{0}\right)=z$,

$$
F^{*}(t, y, z)=V^{*}(t) .
$$

This is the optimal wealth at time $t$, and, hence, the function $F^{*}(t, y, z)$ may be called an optimal wealth function.

The following theorem is the main result which gives a partial differential equation for the optimal wealth function $F^{*}(t, y, z)$ and an expression for the optimal portfolio strategy $\pi^{*}$.

Theorem 8. Given that $\mathscr{M}_{0, t}\left(z_{0}\right)=y$ and $Z_{0, t}\left(z_{0}\right)=z$, suppose that $F^{*}(t, y, z) \in \mathscr{C}^{1,2,2}((0, T) \times(0, \infty) \times(0,1))$. Then the optimal wealth function $F^{*}(t, y, z)$ satisfies the following partial differential equation:

$$
\begin{aligned}
\frac{\partial F^{*}}{\partial t} & +\frac{\partial F^{*}}{\partial z}\left(-a_{1} z+a_{2}(1-z)\right) \\
& +\frac{\partial F^{*}}{\partial y \partial z} \rho\left(\theta_{1} z+\theta_{2}(1-z)\right) y(1-z) z \\
& +\frac{1}{2} \frac{\partial^{2} F^{*}}{\partial y^{2}}\left(\theta_{1} z+\theta_{2}(1-z)\right) y^{2} \\
& +\frac{1}{2} \frac{\partial^{2} F^{*}}{\partial z^{2}} \rho^{2}(1-z)^{2} z^{2}-r F^{*}=0
\end{aligned}
$$

with terminal condition

$$
F^{*}\left(T, \mathscr{M}_{0, T}\left(z_{0}\right), Z_{0, T}\left(z_{0}\right)\right)=V^{*}(T)=\xi .
$$

Furthermore, the optimal portfolio strategy $\pi^{*}(t)$ is given by

$$
\pi^{*}(t)=\left(\frac{\partial F}{\partial y}\left(\theta_{1} z+\theta_{2}(1-z)\right) y+\frac{\partial F}{\partial z} \rho(1-z) z\right) \sigma^{-1} e^{r t}
$$

where

$$
\begin{aligned}
& \frac{\partial F}{\partial y}=\mathscr{M}_{0, t}^{-1}\left(z_{0}\right) E^{\mathbb{Q}}[ \kappa\left(v_{0}\right) \mathscr{M}_{0, T}\left(z_{0}\right) e^{-2 r T} \\
&\left.\times I^{\prime}\left(\kappa\left(v_{0}\right) \mathscr{M}_{0, T}\left(z_{0}\right) e^{-r T}\right) \mid \mathscr{F}(t)\right], \\
& \frac{\partial F}{\partial z}=e^{-r T} E^{\mathbb{Q}}\left[\left(\int_{t}^{T}\left(\theta_{1}-\theta_{2}\right) D_{0, u}\left(z_{0}\right) d \widehat{W}^{\mathbb{Q}}(u)\right)\right. \\
& \cdot\left(I\left(\kappa\left(v_{0}\right) \mathscr{M}_{0, T}\left(z_{0}\right) e^{-r T}\right)\right. \\
&+\mathscr{M}_{0, T}\left(z_{0}\right) \kappa\left(v_{0}\right) e^{-r T} \\
&\left.\left.\times I^{\prime}\left(\kappa\left(v_{0}\right) \mathscr{M}_{0, T}\left(z_{0}\right) e^{-r T}\right)\right) \mid \mathscr{F}(t)\right]
\end{aligned}
$$

$$
\cdot D_{0, t}^{-1}\left(z_{0}\right)
$$

Proof. The results are proved using similar arguments in Elliott et al. [43], for example, Proposition 3.1. Firstly, applying Itô's differentiation rule to $F(t, y, z)$ gives

$$
\begin{aligned}
F(t, y, z)= & F\left(0, y_{0}, z_{0}\right)+\int_{0}^{t} \frac{\partial F}{\partial u} d u+\int_{0}^{t} \frac{\partial F}{\partial y} d \mathscr{M}_{0, u}\left(z_{0}\right) \\
& +\int_{0}^{t} \frac{\partial F}{\partial z} d Z_{0, u}\left(z_{0}\right) \\
& +\frac{1}{2} \int_{0}^{t} \frac{\partial^{2} F}{\partial y^{2}} d\left\langle\mathscr{M}_{0, \cdot}\left(z_{0}\right), \mathscr{M}_{0, \cdot}\left(z_{0}\right)\right\rangle(u) \\
& +\int_{0}^{t} \frac{\partial^{2} F}{\partial y \partial z} d\left\langle\mathscr{M}_{0, \cdot}\left(z_{0}\right), Z_{0, \cdot}\left(z_{0}\right)\right\rangle(u) \\
& +\frac{1}{2} \int_{0}^{t} \frac{\partial^{2} F}{\partial z^{2}} d\left\langle Z_{0, \cdot}\left(z_{0}\right), Z_{0, \cdot}\left(z_{0}\right)\right\rangle(u) .
\end{aligned}
$$

Here $\left\{\left\langle A_{1}, A_{2}\right\rangle(t) \mid t \in \mathscr{T}\right\}$ is the predictable quadratic covariation of the processes $\left\{A_{1}(t) \mid t \in \mathscr{T}\right\}$ and $\left\{A_{2}(t) \mid\right.$ $t \in \mathscr{T}\}$.

Rearranging then gives

$$
\begin{aligned}
& F(t, y, z)= F\left(0, y_{0}, z_{0}\right) \\
&+\int_{0}^{t}\left(\frac{\partial F}{\partial u}+\frac{\partial F}{\partial z}\left(-a_{1} Z_{0, u}\left(z_{0}\right)\right.\right. \\
&\left.+a_{2}\left(1-Z_{0, u}\left(z_{0}\right)\right)\right)
\end{aligned}
$$




$$
\begin{aligned}
&+ \frac{1}{2} \frac{\partial^{2} F}{\partial y^{2}} \Theta_{0, u}^{2}\left(z_{0}\right) \mathscr{M}_{0, u}^{2}\left(z_{0}\right) \\
&+ \frac{1}{2} \frac{\partial^{2} F}{\partial z^{2}} \rho^{2}\left(1-Z_{0, u}\left(z_{0}\right)\right)^{2} Z_{0, u}^{2}\left(z_{0}\right) \\
&+ \frac{\partial^{2} F}{\partial y \partial z} \rho \Theta_{0, u}\left(z_{0}\right) \mathscr{M}_{0, u}\left(z_{0}\right) \\
&+\left.\left(1-Z_{0, u}\left(z_{0}\right)\right) Z_{0, u}\left(z_{0}\right)\right) d u \\
&+\int_{0}^{t}\left(\frac{\partial F}{\partial y} \Theta_{0, u}\left(z_{0}\right) \mathscr{M}_{0, u}\left(z_{0}\right)\right. \\
&+\frac{\partial F}{\partial z} \rho\left(1-Z_{0, u}\left(z_{0}\right)\right) \\
&\left.+Z_{0, u}\left(z_{0}\right)\right) d \widehat{W}^{\mathbb{Q}}(u)
\end{aligned}
$$

From Lemma 7 and the martingale representation for the discounted optimal wealth described in Section 3,

$$
F(t, y, z)=e^{-r t} V^{*}(t)=v_{0}+\int_{0}^{t} \psi(u) d \widehat{W}^{\mathbb{Q}}(u), \quad \mathbb{P} \text {-a.s. }
$$

Consequently, $\{F(t, y, z) \mid t \in \mathscr{T}\}$ is an $(\mathbb{F}, \mathbb{Q})$-(local)martingale, and, hence, it must be an $(\mathbb{F}, \mathbb{Q})$-special semimartingale.

By the unique decomposition of a special semimartingale, the predictable term of finite variation in (64) must be indistinguishable from the zero process. Consequently,

$$
\begin{aligned}
\frac{\partial F}{\partial t}+ & \frac{\partial F}{\partial z}\left(-a_{1} Z_{0, t}\left(z_{0}\right)+a_{2}\left(1-Z_{0, t}\left(z_{0}\right)\right)\right) \\
& +\frac{\partial^{2} F}{\partial y \partial z} \rho \Theta_{0, t}\left(z_{0}\right) \mathscr{M}_{0, t}\left(z_{0}\right)\left(1-Z_{0, t}\left(z_{0}\right)\right) Z_{0, t}\left(z_{0}\right) \\
& +\frac{1}{2} \frac{\partial^{2} F}{\partial y^{2}} \Theta_{0, t}^{2}\left(z_{0}\right) \mathscr{M}_{0, t}^{2}\left(z_{0}\right) \\
& +\frac{1}{2} \frac{\partial^{2} F}{\partial z^{2}} \rho^{2}\left(1-Z_{0, t}\left(z_{0}\right)\right)^{2} Z_{0, t}^{2}\left(z_{0}\right)=0
\end{aligned}
$$

Then

$$
\begin{aligned}
\frac{\partial F}{\partial t}+ & \frac{\partial F}{\partial z}\left(-a_{1} z+a_{2}(1-z)\right) \\
& +\frac{\partial^{2} F}{\partial y \partial z} \rho\left(\theta_{1} z+\theta_{2}(1-z)\right) y(1-z) z \\
& +\frac{1}{2} \frac{\partial^{2} F}{\partial y^{2}}\left(\theta_{1} z+\theta_{2}(1-z)\right)^{2} y^{2} \\
& +\frac{1}{2} \frac{\partial^{2} F}{\partial z^{2}} \rho^{2}(1-z)^{2} z^{2}=0 .
\end{aligned}
$$

Therefore, (59) follows by noting that $F^{*}(t, y, z)=F(t, y, z) /$ $e^{-r t}$ and by differentiation.

Again by the unique decomposition of a special semimartingale, the integrand processes of the stochastic integrals in (64) and (65) must be indistinguishable. Consequently,

$$
\psi(t)=\frac{\partial F}{\partial y}\left(\theta_{1} z+\theta_{2}(1-z)\right) y+\frac{\partial F}{\partial z} \rho(1-z) z .
$$

Differentiating with respect to $y$ inside the expectation and using a version of Bayes' rule give

$$
\begin{aligned}
\frac{\partial F}{\partial y}=E\left[\kappa\left(v_{0}\right) \mathscr{M}_{t, T}^{2}(z) e^{-2 r T}\right. & \\
& \left.\times I^{\prime}\left(\kappa\left(v_{0}\right) y \mathscr{M}_{t, T}(z) e^{-r T}\right) \mid \mathscr{F}(t)\right] \\
=\mathscr{M}_{0, t}^{-1}\left(z_{0}\right) E^{\mathbb{Q}}\left[\kappa\left(v_{0}\right) \mathscr{M}_{0, T}\left(z_{0}\right) e^{-2 r T}\right. & \\
& \left.\times I^{\prime}\left(\kappa\left(v_{0}\right) \mathscr{M}_{0, T}\left(z_{0}\right) e^{-r T}\right) \mid \mathscr{F}(t)\right] .
\end{aligned}
$$

Furthermore,

$$
\begin{gathered}
\frac{\partial F}{\partial z}=E\left[\frac{\partial \mathscr{M}_{t, T}(z)}{\partial z} e^{-r T} I\left(\kappa\left(v_{0}\right) y \mathscr{M}_{t, T}(z) e^{-r T}\right) \mid \mathscr{F}(t)\right] \\
+E\left[\mathscr{M}_{t, T}(z) e^{-r T} I^{\prime}\left(\kappa\left(v_{0}\right) y \mathscr{M}_{t, T}(z) e^{-r T}\right)\right. \\
\left.\cdot \kappa\left(v_{0}\right) y e^{-r T} \frac{\partial \mathscr{M}_{t, T}(z)}{\partial z} \mid \mathscr{F}(t)\right] .
\end{gathered}
$$

Differentiating with respect to $z$ inside the expectation and using a version of Bayes' rule and Lemma 6 give

$$
\begin{aligned}
\frac{\partial F}{\partial z}=E^{\mathbb{Q}}\left[e^{-r T}\left(\int_{t}^{T}\left(\theta_{1}-\theta_{2}\right) D_{t, u}(z) d \widehat{W}^{\mathbb{Q}}(u)\right)\right. \\
\left.\cdot I\left(\kappa\left(v_{0}\right) \mathscr{M}_{0, T}\left(z_{0}\right) e^{-r T}\right) \mid \mathscr{F}(t)\right] \\
+E^{\mathbb{Q}}\left[I^{\prime}\left(\kappa\left(v_{0}\right) y \mathscr{M}_{t, T}(z) e^{-r T}\right) \kappa\left(v_{0}\right) y e^{-2 r T} \mathscr{M}_{t, T}(z)\right. \\
\left.\times\left(\int_{t}^{T}\left(\theta_{1}-\theta_{2}\right) D_{t, u}(z) d \widehat{W}^{\mathbb{Q}}(u)\right) \mid \mathscr{F}(t)\right] .
\end{aligned}
$$

Consequently,

$$
\begin{aligned}
\frac{\partial F}{\partial z}=E^{\mathbb{Q}}\left[e^{-r T}\left(\int_{t}^{T}\left(\theta_{1}-\theta_{2}\right) D_{0, u}\left(z_{0}\right) d \widehat{W}^{\mathbb{Q}}(u)\right)\right. \\
\left.\cdot I\left(\kappa\left(v_{0}\right) \mathscr{M}_{0, T}\left(z_{0}\right) e^{-r T}\right) \mid \mathscr{F}(t)\right] D_{0, t}^{-1}\left(z_{0}\right)
\end{aligned}
$$




$$
\begin{aligned}
+E^{\mathbb{Q}}[ & \left(\mathscr{M}_{0, T}\left(z_{0}\right) \int_{t}^{T}\left(\theta_{1}-\theta_{2}\right) D_{0, u}\left(z_{0}\right) d \widehat{W}^{\mathbb{Q}}(u)\right) \\
& \cdot \kappa\left(v_{0}\right) e^{-2 r T} \\
& \left.\times I^{\prime}\left(\kappa\left(v_{0}\right) \mathscr{M}_{0, T}\left(z_{0}\right) e^{-r T}\right) \mid \mathscr{F}(t)\right] D_{0, t}^{-1}\left(z_{0}\right) .
\end{aligned}
$$

Expression (61) for the optimal portfolio then follows by noting that

$$
\pi^{*}(t)=\psi(t) \sigma^{-1} e^{r t}
$$

Remark 9. The partial differential equation for the optimal wealth function arising from the two-state situation is neater than that from a general $N$-state case (The author would like to thank the referee for pointing out this.). The former may also have some more numerical advantages than the latter. This may represent an advantage of considering the two-state situation.

Remark 10. It is known that the martingale approach to optimal asset allocation and the risk-neutral pricing and hedging of a contingent claim are related to each other. The partial differential equation for the optimal wealth function may provide some further insights into the link between them. In particular, if the optimal terminal wealth $\xi$ is considered the terminal payoff of the claim, the optimal wealth function may be considered the value function of a self-financing replicating portfolio of the claim and hence the price function of the claim under the no-arbitrage principle. In this case, the partial differential equation for the optimal wealth function may be interpreted as the partial differential equation for the price function of the claim.

Remark 11. There are some advantages of using the stochastic flows approach comparing to the Malliavin calculus approach and the Hamilton-Jacobi-Bellman (HJB) dynamic programming approach. Compared with the Malliavin calculus approach, the stochastic flows approach does not involve the use of infinite-dimensional calculus and may be more intuitively appealing than the Malliavin calculus approach. This advantage of the stochastic flows approach in a general context has also been discussed in, for example, Elliott and Kohlmann [47, 48], amongst others. Furthermore, the stochastic flows approach gives rise to a partial differential equation for the optimal wealth function, whereas this result may not be obtained using the Malliavin calculus approach. Of course, the Malliavin calculus approach does have some advantages over the stochastic flows approach. One advantage is that the former can be used in the non-Markovian situation while the latter can only be used in the Markovian situation. In this sense, the Malliavin calculus approach is more general than the stochastic flows approach. Nevertheless, in the filtered market considered here, the wealth process and the state probability process are jointly Markovian with respect to the observed filtration, so the stochastic flows approach is applicable in the current set up. Compared with the HJB dynamic programming approach, the stochastic flows approach provides a way to justify some differentiability conditions. In particular, some differentiability conditions for the optimal wealth function in Theorem 8 may be justified by the stochastic flows of diffeomorphisms, whereas the HJB dynamic programming approach itself may not be used to justify some differentiability conditions required in the verification theorem and viscosity solutions to the $\mathrm{HJB}$ equation may be required. Furthermore, the verification theorem arising from the HJB dynamic programming approach requires the uniform integrability condition for the value function, which may be uneasy to verify in some situations. Using the stochastic flows approach, the optimal portfolio process depends on the optimal wealth function instead of the value function. Consequently, the verification of the uniform integrability of the value function is not required in the stochastic flows approach. (The author would like to thank the referee for stimulating the discussion.)

\section{Conclusions}

An approach based on a martingale representation and stochastic flows of diffeomorphisms was adopted to discuss an optimal asset allocation problem in a simple two-state Markovian regime-switching market. This approach may be able to accommodate quite a general class of utility functions under some differentiability conditions. As usual, standard filtering theory was first used to transform the original market into one with complete observations, where the latter is complete. When the values of the underlying state variables were given, using stochastic flows of diffeomorphisms for the filtering equation and the unique decomposition of a special semimartingale, the integrand of a martingale representation for the discounted optimal wealth process was identified which gave rise to an optimal portfolio strategy. A partial differential equation for the optimal wealth function was also provided. The results may be generalized to the case of an $\mathrm{N}$-state hidden Markov chain though some complicated notation may be involved.

The approach considered here may pave a way for some potentially interesting topics for future research. Firstly, one may consider incorporating portfolio constraints in the optimal asset allocation problem. Secondly, one may extend the current model to a Markovian regime-switching jumpdiffusion model. Thirdly, as relating to the second topic, one may consider the application of the approach to study an optimal investment problem of an insurer. In this case, one needs to take into account insurance liabilities which may be modeled by Markov-modulated compound Poisson processes. The papers by Elliott and Siu [21] and Siu [26] may provide some clues along this direction.

\section{Conflict of Interests}

The author declares that there is no conflict of interests regarding the publication of this paper. 


\section{Acknowledgments}

The author would like to thank the referee for helpful comments. The author also wishes to thank the Australian Research Council.

\section{References}

[1] H. M. Markowitz, "Portfolio selection," Journal of Finance, vol. 7, no. 1, pp. 77-91, 1952.

[2] R. C. Merton, "Lifetime portfolio selection under uncertainty: the continuous-time model," The Review of Economics and Statistics, vol. 51, no. 3, pp. 247-257, 1969.

[3] R. C. Merton, "Optimum consumption and portfolio rules in a continuous-time model," Journal of Economic Theory, vol. 3, no. 4, pp. 373-413, 1971.

[4] S. R. Pliska, "A stochastic calculus model of continuous trading: optimal portfolios," Mathematics of Operations Research, vol. 11, no. 2, pp. 371-384, 1986.

[5] I. Karatzas, J. P. Lehoczky, and S. E. Shreve, "Optimal portfolio and consumption decisions for a 'small investor' on a finite horizon," SIAM Journal on Control and Optimization, vol. 25, no. 6, pp. 1557-1586, 1987.

[6] J. C. Cox and C.-F. Huang, "Optimal consumption and portfolio policies when asset prices follow a diffusion process," Journal of Economic Theory, vol. 49, no. 1, pp. 33-83, 1989.

[7] J. Cvitanic and I. Karatzas, "Convex duality in constrained portfolio optimization," The Annals of Applied Probability, vol. 2, no. 4, pp. 767-818, 1992.

[8] I. Karatzas and S. E. Shreve, Methods of Mathematical Finance, Springer, New York, NY, USA, 1998.

[9] R. J. Elliott and P. E. Kopp, Mathematics of Financial Markets, Springer, New York, NY, USA, 2nd edition, 2005.

[10] H. Pham, Continuous-time Stochastic Control and Optimization with Financial Applications, Springer, New York, NY, USA, 2009.

[11] H. U. Gerber and E. S. Shiu, "Investing for retirement: optimal capital growth and dynamic asset allocation," North American Actuarial Journal, vol. 4, no. 2, pp. 42-62, 2000.

[12] P. P. Boyle, "Discussion on 'investing for retirement: optimal capital growth and dynamic asset allocation by H. U. Gerber and E. S. W. Shiu," North American Actuarial Journal, vol. 4, no. 2, pp. 58-59, 2000.

[13] X. Y. Zhou and G. Yin, "Markowitz's mean-variance portfolio selection with regime switching: a continuous-time model," SIAM Journal on Control and Optimization, vol. 42, no. 4, pp. 1466-1482, 2003.

[14] G. Yin and X. Y. Zhou, "Markowitz's mean-variance portfolio selection with regime switching: from discrete-time models to their continuous-time limits," IEEE Transactions on Automatic Control, vol. 49, no. 3, pp. 349-360, 2004.

[15] J. Sass and U. G. Haussmann, "Optimizing the terminal wealth under partial information: the drift process as a continuous time Markov chain," Finance and Stochastics, vol. 8, no. 4, pp. 553577, 2004.

[16] N. Baeuerle and U. Rieder, "Portfolio optimization with jumps and unobservable intensity process," Mathematical Finance, vol. 17, no. 2, pp. 205-224, 2007.

[17] B.-G. Jang, H. Keun Koo, H. Liu, and M. Loewenstein, "Liquidity premia and transaction costs," The Journal of Finance, vol. 62, no. 5, pp. 2329-2366, 2007.
[18] H. Nagai and W. J. Runggaldier, "PDE approach to utility maximization for market models with hidden Markov factors," in Seminar on Stochastic Analysis, Random Fields and Applications $V$, R. C. Dalang, M. Dozzi, and F. Russo, Eds., vol. 59 of Progress in Probability, pp. 493-506, Birkhäuser, Basel, Switzerland, 2008.

[19] L. R. Sotomayor and A. Cadenillas, "Explicit solutions of consumption-investment problems in financial markets with regime switching," Mathematical Finance, vol. 19, no. 2, pp. 251279, 2009.

[20] X. Zhang, T. K. Siu, and Q. Meng, "Portfolio selection in the enlarged Markovian regime-switching market," SIAM Journal on Control and Optimization, vol. 48, no. 5, pp. 3368-3388, 2009/10.

[21] R. J. Elliott and T. K. Siu, "An HMM approach for optimal investment of an insurer," International Journal of Robust and Nonlinear Control, vol. 22, no. 7, pp. 778-807, 2012.

[22] R. J. Elliott, T. K. Siu, and A. Badescu, "On mean-variance portfolio selection under a hidden Markovian regime-switching model," Economic Modelling, vol. 27, no. 3, pp. 678-686, 2010.

[23] R. Korn, T. K. Siu, and A. Zhang, "Asset allocation for a DC pension fund under regime switching environment," European Actuarial Journal, vol. 1, no. 2, supplement, pp. 361-377, 2011.

[24] T. K. Siu, "Long-term strategic asset allocation with inflation risk and regime switching," Quantitative Finance, vol. 11, no. 10, pp. 1565-1580, 2011.

[25] T. K. Siu, "A BSDE approach to risk-based asset allocation of pension funds with regime switching," Annals of Operations Research, vol. 201, no. 1, pp. 449-473, 2012.

[26] T. K. Siu, "A BSDE approach to optimal investment of an insurer with hidden regime switching," Stochastic Analysis and Applications, vol. 31, no. 1, pp. 1-18, 2013.

[27] Y. Shen and T. K. Siu, "Asset allocation under stochastic interest rate with regime switching," Economic Modelling, vol. 29, no. 4, pp. 1126-1136, 2012.

[28] S. J. Taylor, "Markov processes and the distribution of volatility: a comparison of discrete and continuous specifications," Philosophical Transactions of the Royal Society of London: Series A, vol. 357, pp. 2059-2070, 1999.

[29] S. J. Taylor, Asset Price Dynamics, Volatility, and Prediction, Princeton University Press, Princeton, NJ, USA, 2005.

[30] J. Wei, R. Wang, and H. Yang, "Optimal surrender strategies for equity-indexed annuity investors with partial information," Statistics and Probability Letters, vol. 82, no. 7, pp. 1251-1258, 2012.

[31] R. J. Elliott, L. Aggoun, and J. B. Moore, Hidden Markov Models: Estimation and Control, Springer, Berlin, Germany, 1994.

[32] S. J. Taylor, Modelling Financial Time Series, John Wiley and Sons, Chichester, UK, 1986.

[33] X. Guo, "Information and option pricings," Quantitative Finance, vol. 1, no. 1, pp. 38-44, 2001.

[34] H. U. Gerber and E. S. W. Shiu, "Discussion on 'asset allocation with hedge funds on the menu' by Boyle, P. P. and Liew, S. S., October 2007," North American Actuarial Journal, vol. 12, no. 1, pp. 89-90, 2009.

[35] R. Lipster and A. N. Shiryaev, Statistics of Random Processes, Springer, Berlin, Germany, 2003.

[36] R. J. Elliott, Stochastic Calculus and Applications, Springer, New York, NY, USA, 1982.

[37] R. Rishel, "Necessary and sufficient dynamic programming conditions for continuous time stochastic optimal control," 
SIAM Journal on Control and Optimization, vol. 8, no. 4, pp. 559-571, 1970.

[38] T. Duncan and P. Varaiya, "On the solutions of a stochastic control system," SIAM Journal on Control and Optimization, vol. 9, pp. 354-371, 1971.

[39] T. Duncan and P. Varaiya, "On the solutions of a stochastic control system. II," SIAM Journal on Control and Optimization, vol. 13, no. 5, pp. 1077-1092, 1975.

[40] M. H. Davis, "On the existence of optimal policies in stochastic control," SIAM Journal on Control and Optimization, vol. 11, no. 4, pp. 587-594, 1973.

[41] D. B. Colwell, R. J. Elliott, and P. . Kopp, "Martingale representation and hedging policies," Stochastic Processes and their Applications, vol. 38, no. 2, pp. 335-345, 1991.

[42] D. B. Colwell and R. J. Elliott, "Discontinuous asset prices and nonattainable contingent claims," Mathematical Finance, vol. 3, no. 3, pp. 295-308, 1993.

[43] R. J. Elliott, T. K. Siu, and H. Yang, "Martingale representation for contingent claims with regime switching," Communications on Stochastic Analysis, vol. 1, no. 2, pp. 279-292, 2007.

[44] H. Kunita, "Stochastic partial differential equations connected with nonlinear filtering," in Nonlinear Filtering and Stochastic Control, vol. 972 of Lecture Notes in Mathematics, pp. 100-169, Springer, New York, NY, USA, 1982.

[45] H. Kunita, "Stochastic differential equations and stochastic flows of diffeomorphisms," in École d'Été de Probabilités de Saint-Flour XII-1982, vol. 1097 of Lecture Notes in Mathematics, pp. 143-303, Springer, New York, NY, USA, 1984.

[46] J. M. Bismut, "Martingales, the Malliavin calculus and hypoellipticity under general Hörmander's conditions," Zeitschrift für Wahrscheinlichkeitstheorie und Verwandte Gebiete, vol. 56, no. 4, pp. 469-505, 1981.

[47] R. J. Elliott and M. Kohlmann, "Martingale representation and the Malliavin calculus," Applied Mathematics and Optimization, vol. 20, no. 1, pp. 105-112, 1989.

[48] R. J. Elliott and M. Kohlmann, "Integration by parts, homogeneous chaos expansions and smooth densities," The Annals of Probability, vol. 17, no. 1, pp. 194-207, 1989. 


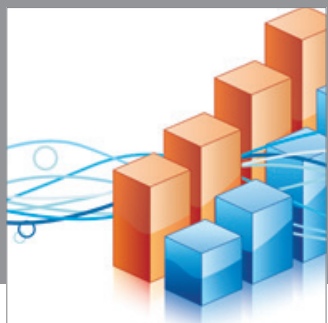

Advances in

Operations Research

mansans

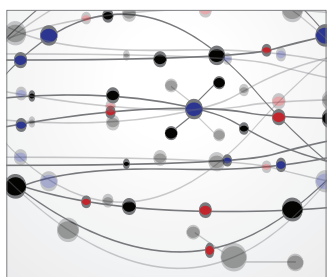

The Scientific World Journal
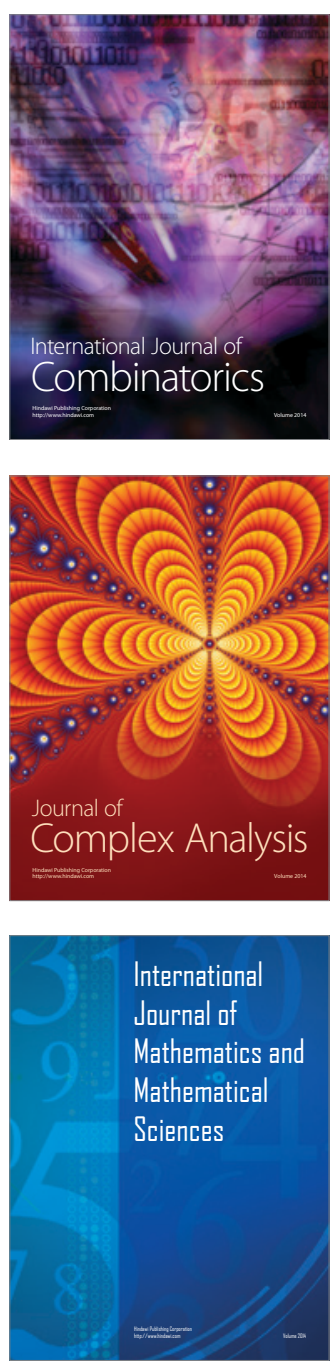
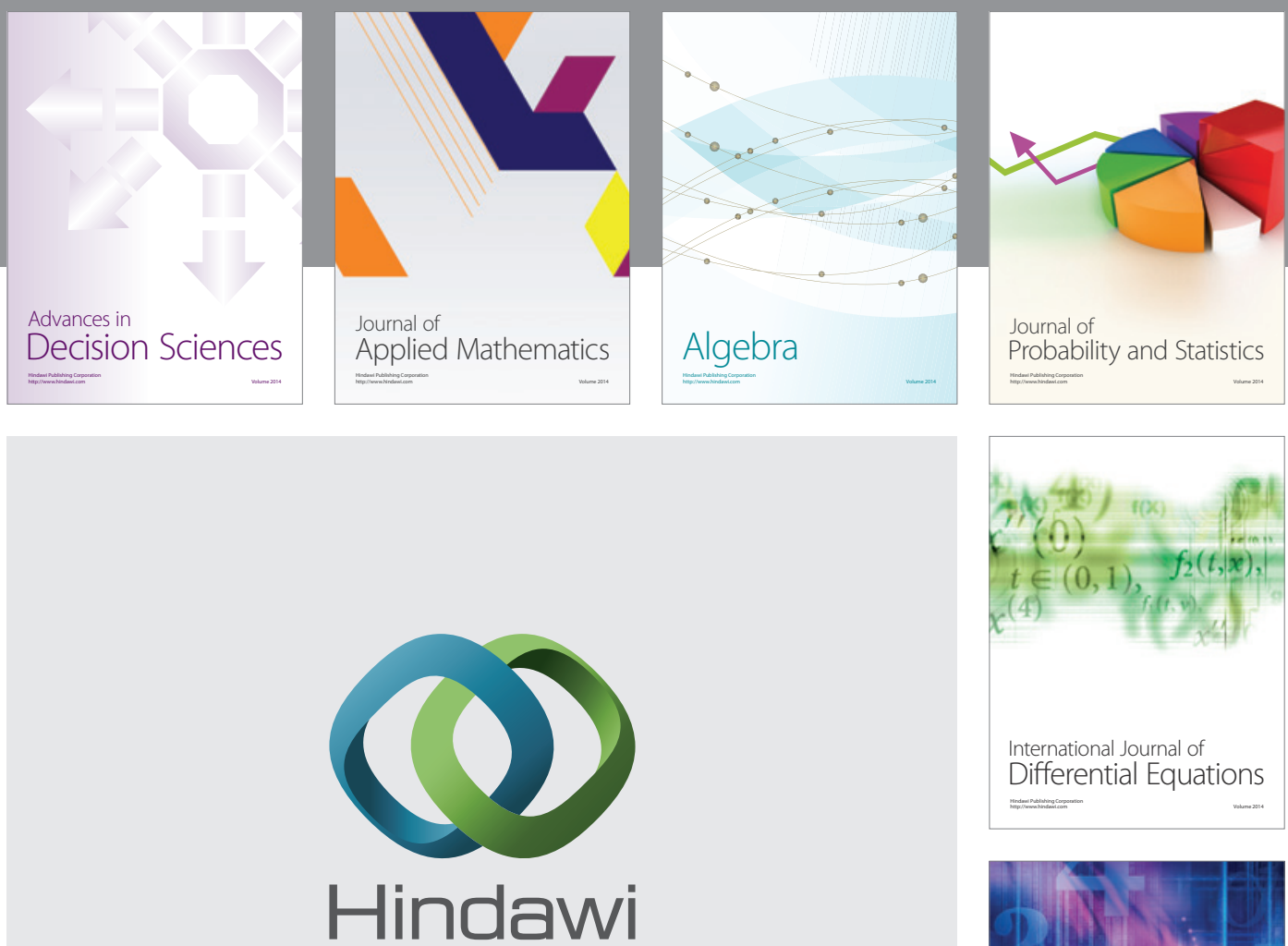

Submit your manuscripts at http://www.hindawi.com
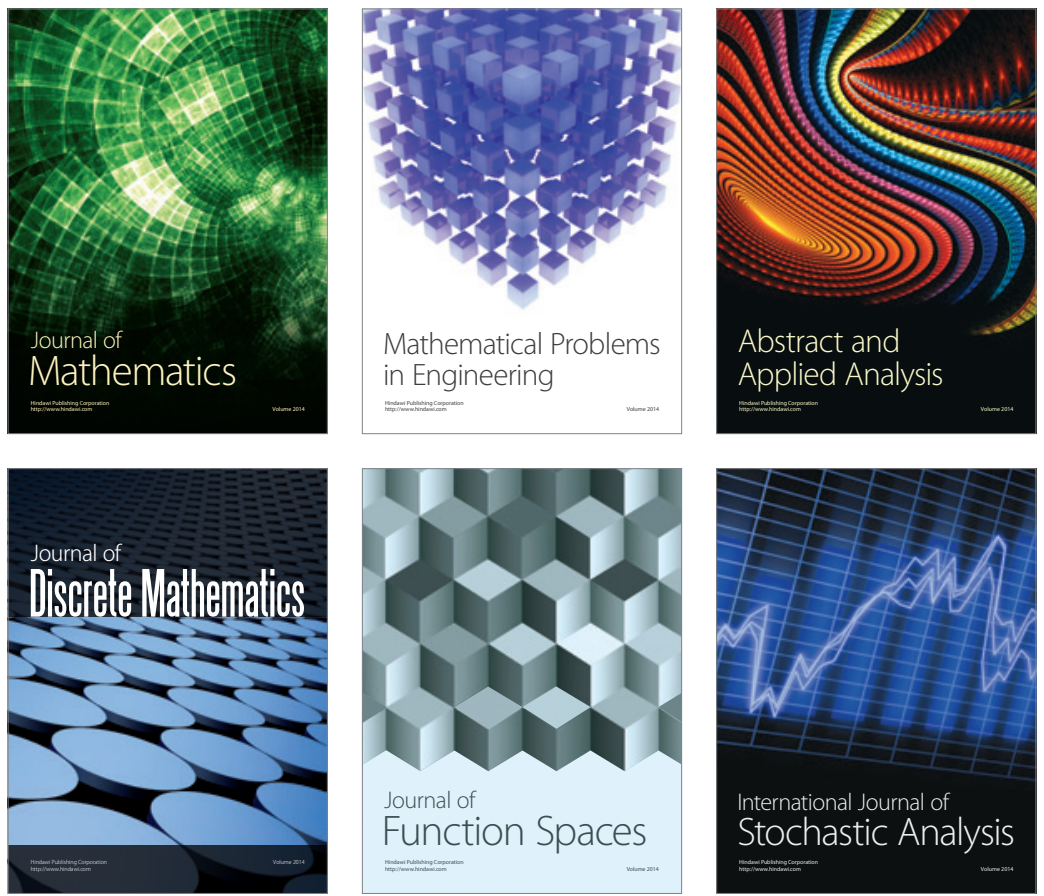

Journal of

Function Spaces

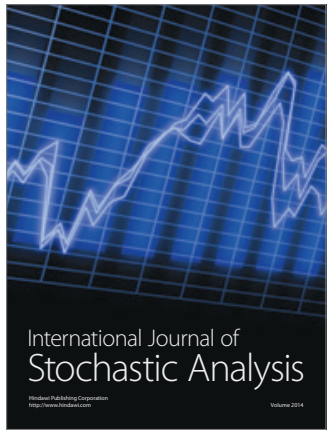

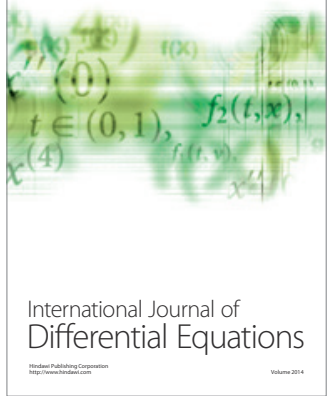
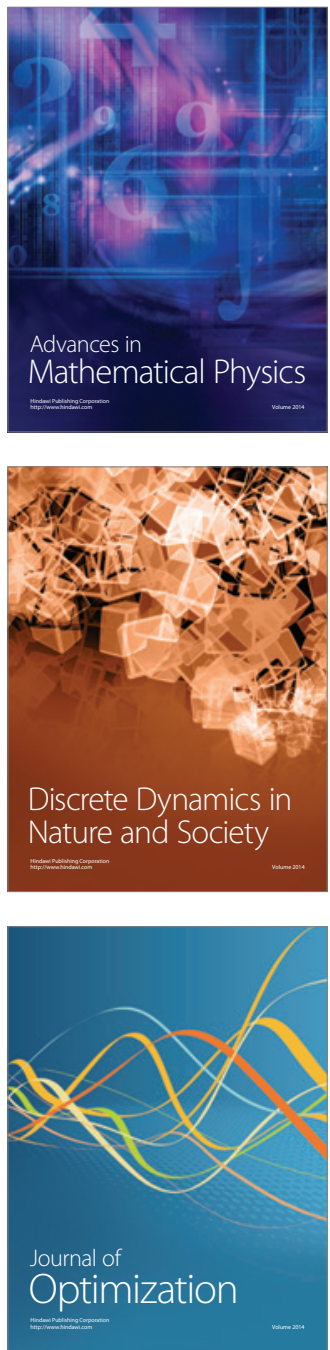